
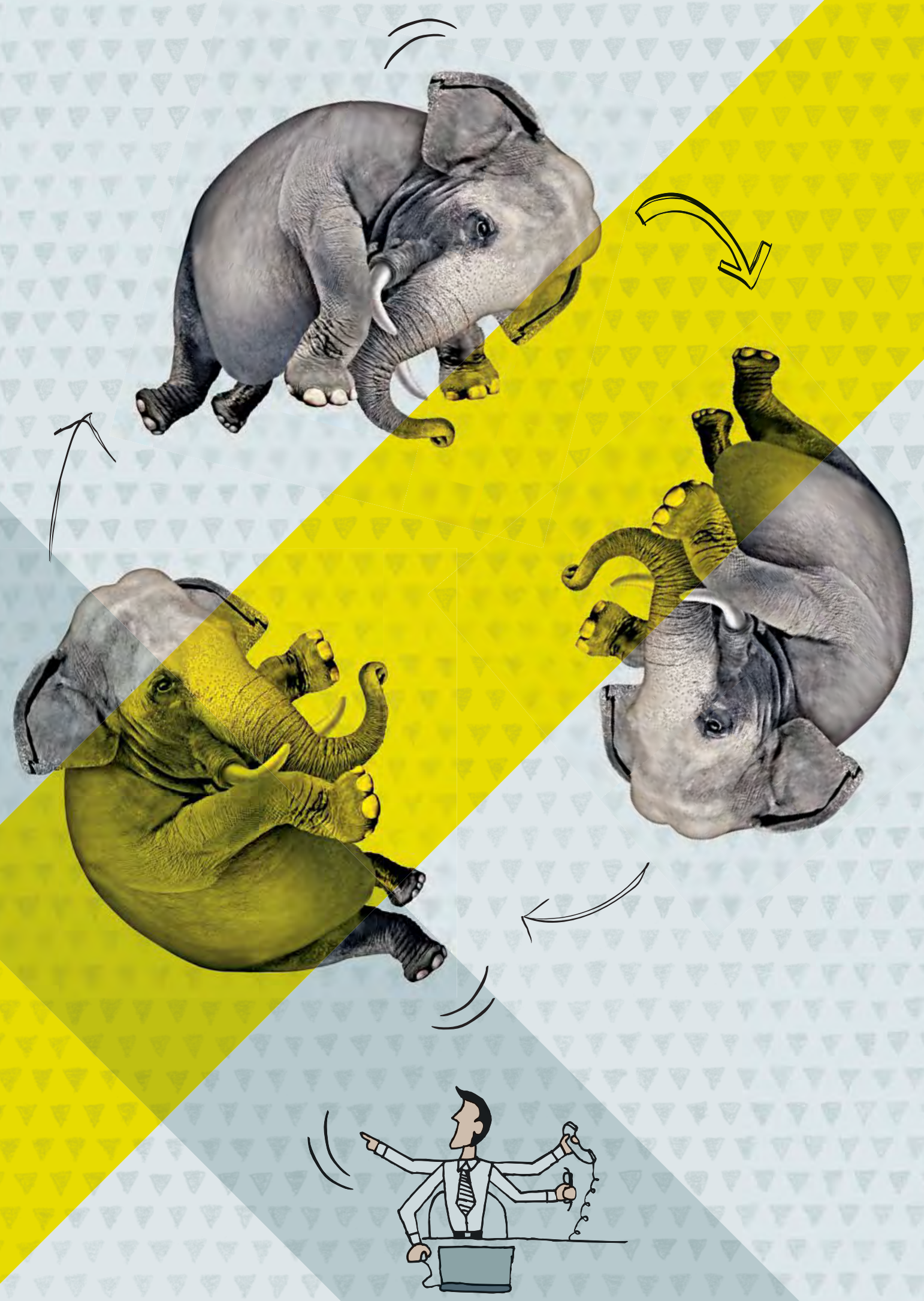


\title{
Stressed Out: How Stress Develops and How to Cope with It
}

\author{
Marcello Mortillaro and Klaus R. Scherer
}

\section{KEYWORDS}

Stress, Burnout, Stress Measurement, Coping with Stress

\section{THE AUTHORS}

Marcello Mortillaro,

Senior Researcher, Marcello.Mortillaro@unige.ch

Klaus R. Scherer, Professor and Founding Director, Klaus.Scherer@unige.ch

\section{Both}

of the Swiss Centre for Affective Sciences,

University of Geneva, Switzerland

http://www.affective-sciences.org
Managers - whether working in marketing or not - increasingly suffer from stress. Burnout has become a widespread condition; even stress-related suicides are not unheard of. Yet stress can also be positive, spurring us on to tackle challenging situations. Reason enough to take a closer look at how stress develops and how we can best cope with it.

Stress as an emotion /// The word "stress" is used to refer to many different experiences caused by many different situations. It is sometimes described as a psychological phenomenon, sometimes as a physiological state. In fact, attempting to separate the two is misguided: Like all emotions, stress has both a psychological and a physiological component. Stress is not some special syndrome, but the result of an intense emotional state that persists for an extended period of time. In this respect it differs from most emotions, which are short episodes.

Behind most stress responses lies a situation that elicits an emotion. If the individual cannot cope with this situation, or the situation lasts too long or becomes more intense, the emotion can turn into "emotion-stress". For example, people normally feel apprehensive before important meetings with clients. But if they experience this anxiety continuously over many days, it can become "anxiety-stress". 
FIGURE 1:

\section{Stress level and individual performance}

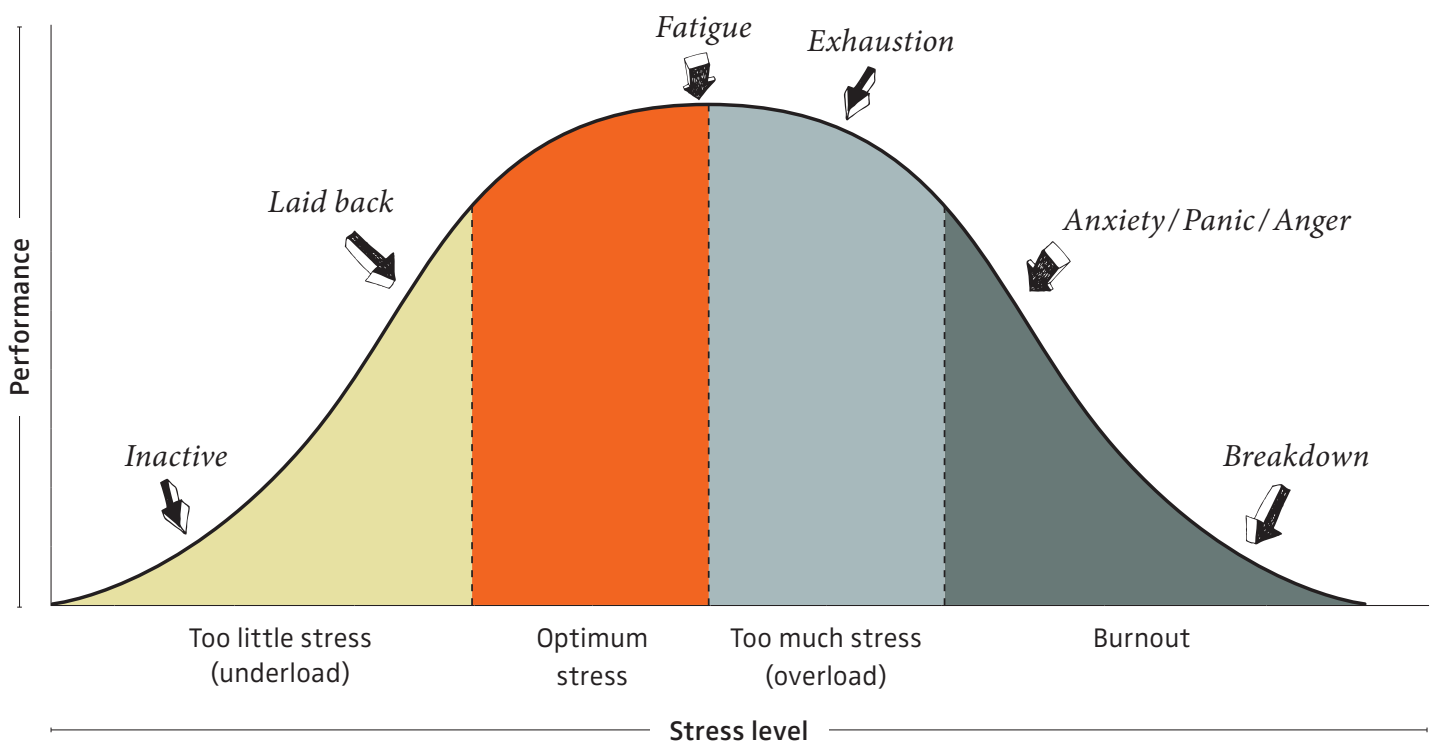

Characteristics of stress as an emotion /// So, before we delve further into the nature of stress, we need to establish what emotions are and what this means for stress in particular. Emotions concern objects that are relevant for the individual. If the individual experiences an emotion, then the stimulus that caused the reaction is important for that individual. For instance, an important meeting with a client is more likely to elicit stress than a routine team meeting would.

Emotions have evolved because they help us cope with challenges. For example, anger triggers the body's resources for a physical fight. Signs of anger, such as facial expressions and voice quality, have the potential to discourage aggressors. A stress response, if it does not become too strong, can give us the energy to cope with a difficult situation. The stress felt by someone giving a presentation before a large audience, for instance, can make him or her more focused and alert (Figure 1).
Emotions trigger reactions in our various bodily systems. The stress response is also accompanied by strong physiological changes; it has a pervasive effect on our organism and on functions such as memory and learning.

Emotions have "control precedence": They take priority when it comes to controlling behavior and experience. This is the main reason why emotions influence our judgment, decisions and behavior.

Stressful feelings are subjective evaluations /// According to the appraisal theory of emotions, our experience of stress depends entirely on how we evaluate the circumstances impacting our individual well-being. In principle, any event can be a stressor: What matters is our subjective evaluation of the event, its relevance for us. Certain events can produce complex emotional states, such as a mixture of anger and worry. If this emotional state is prolonged, it can lead to stress. 


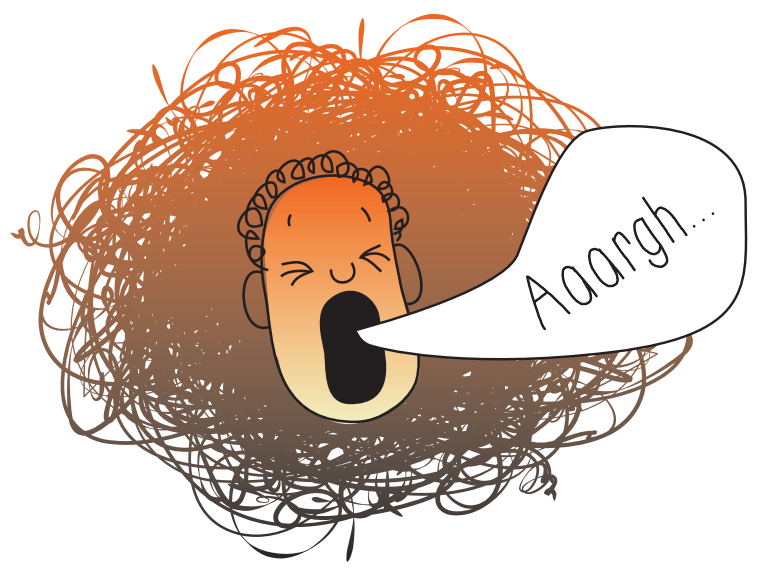

A single situation can be a powerful stressor for one person yet entirely irrelevant for another. For example, soccer fans watching their team play react differently than someone who is not interested in soccer does when watching a game. Situation appraisal is continuous: We are constantly scanning and evaluating what is going on around us to see whether the stimuli are relevant, what their implications for us are, how well we can cope with them and what their normative significance is for our self-image, values and moral rules.

Identifying stress - what the voice reveals /// Stressful situations have a direct effect on different components of our emotional experience: our physiology, "action tendency", expression and subjective feelings. There has been a significant amount of research into expressive components such as facial expression and signs of stress in the voice. Indeed, the voice is particularly sensitive to stress.

The two major determinants of vocalization are respiration and muscle tone, both of which are heavily influenced by emotions and stress. More than 30 years ago, using primitive technological equipment, researchers already noted how the tension caused by emotions and stress is reflected in the sound of the voice.

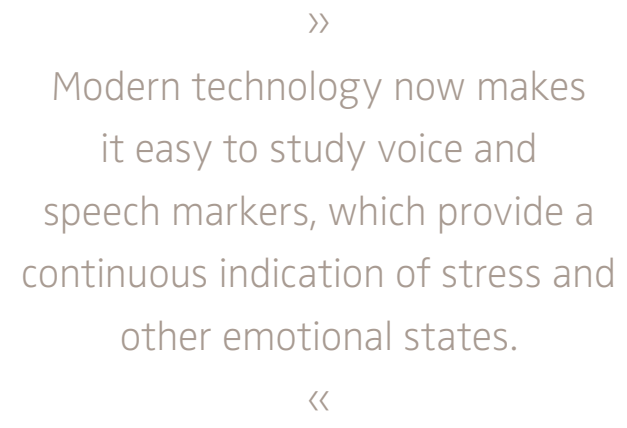

One of the most interesting vocal parameters is fundamental frequency, commonly described in terms of pitch. Research shows that stress is often associated with an increase in fundamental frequency, although there are many individual differences linked to personality. Other parameters also change, such as volume and energy distribution across the spectrum. Modern technology now makes it easy to study voice and speech markers, which provide a continuous indication of stress and other emotional states.

Coping with stress /// Consider the following example: Two people working for a company are told to carry out a task that is critical to the future of the company. The first person sees the task as a challenge that she can cope with: She believes she has the necessary personal resources and skills and is proud to have been chosen for the task. Her colleague, however, sees the task as a threat: He is afraid that he doesn't have the necessary personal resources or skills and is very worried about having been chosen for the task. If this emotional state persists over the following days, he will experience a strong negative feeling of stress. While the first person is likely to experience positive stress - stress that gives her additional energy to tackle the task, the second person has a problem. But what can he actually do to reduce his feelings of stress? 
FIGURE 2:

\section{Three dimensions of coping strategies}

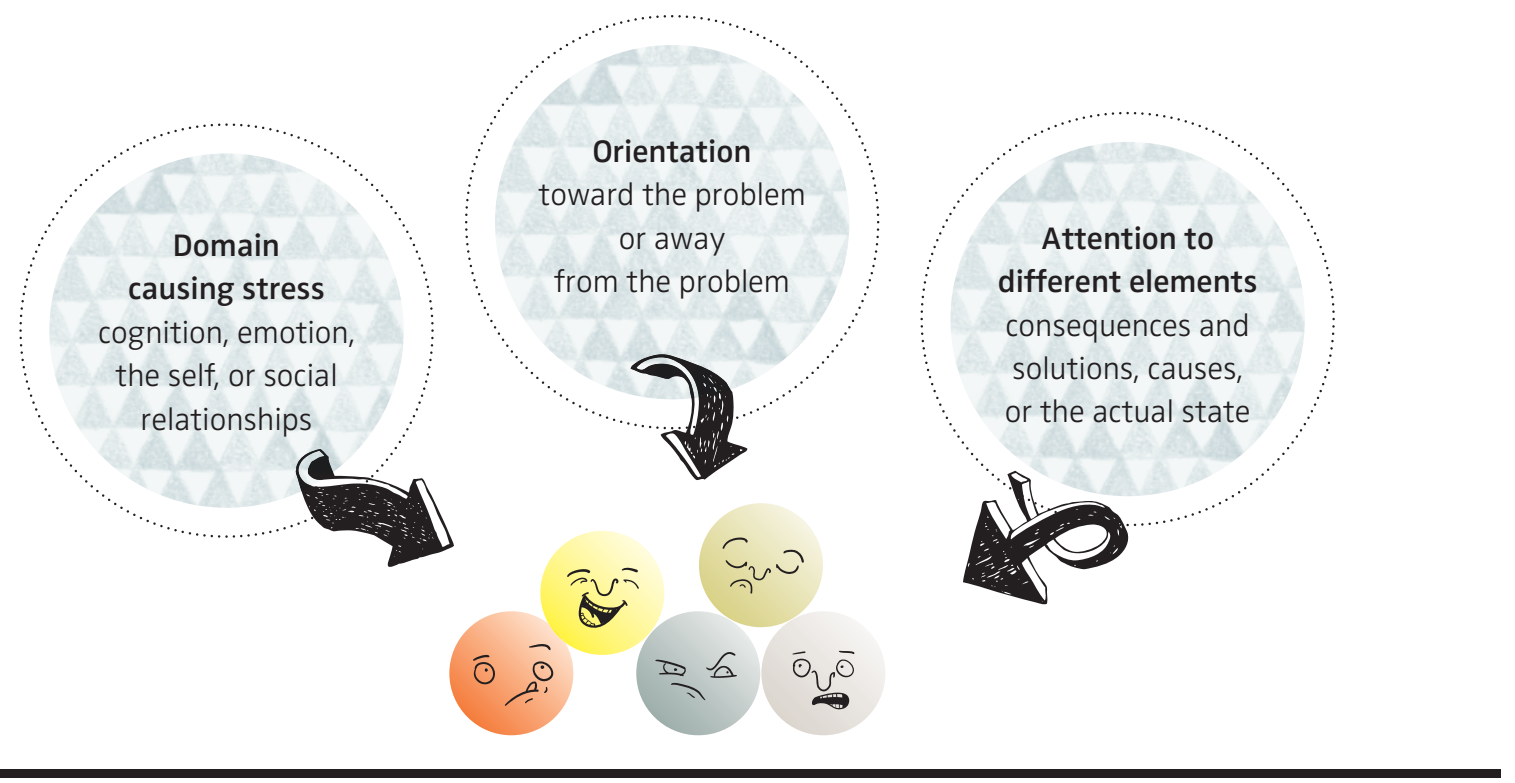

\author{
〉) \\ In principle, any event \\ can be a stressor: What matters \\ is our subjective \\ evaluation of the event.
}

《
Of course, no one-size-fits-all solution exists. The effectiveness or "functionality" of a strategy depends on a person's coping potential - their perceived ability to cope with a situation. The individual, the situation and the concrete social context in which the stressor occurs are all crucial for choosing a response.

Psychologists use the term "coping processes" to refer to the concrete strategies that individuals use to handle such events and their consequences. Coping strategies have three dimensions (Figure 2). These different dimensions also provide the key to understanding how we can best cope with stress.

It should be noted that the three dimensions are not strategies in themselves; they describe possible reactions by individuals. Coping strategies are typically focused on more than one dimension. In our example of the two colleagues assigned a challenging task, the second person might try to change the situation by seeking support or advice on how to perform the 
task successfully: This is a "domain-focused" coping strategy that is oriented toward the problem. Alternatively, he may change his personal goals or values, telling himself: "Well, my career is not that important to me and I want a new job anyway." This coping strategy is focused on elements other than the actual domain, and is oriented away from the problem.

Another form of coping strategy is rumination: the act of continuously thinking about the event and its causes. Rumination addresses cognitive aspects of the stressful situation, is oriented toward the problem, and focuses on the actual situation and its causes. A contrasting strategy is emotion suppression, which aims to regulate the emotion by adopting an avoidance attitude toward the actual problem and its causes.

These different strategies are not mutually exclusive; often, people use multiple strategies at the same time. Not all coping strategies are good for individual well-being or favor a cooperation-based work culture, either. For instance, people with lower levels of emotional competence tend to use more dysfunctional coping strategies, often with a negative effect on their emotional stability. If they are managers, they tend to over-externalize or blame others for failures. They also tend to use other dysfunctional strategies such as wishful thinking, bolstering self-esteem and substance abuse. Such managers more frequently experience worry, show lower emotional stability and demonstrate fewer positive work values, such as the value of intellectual challenge.

\section{How can companies reduce stress for their employees?}

/// One option is to organize training sessions that actively address emotional competence and stress management. They may soon also be able to use new technology to identify staff suffering from stress: Different research groups are currently working on software applications that can detect individual stress levels via cellphone by analyzing vocal characteristics. Companies could use these applications as a tool for immediately managing workplace health, either giving stressed salespersons standard recommendations for reducing stress or by having trainers personally coach them over the phone about how to deal with stress. Although, of course, the problem remains that it may be the cell phones that are causing them stress in the first place...

I.

\section{FURTHER READING}

Scherer, K. R. (1986):

"Voice, stress, and emotion", in M.H. Appley, \& R. Trumbull (eds), Dynamics of stress, pp. 159 - 181. New York: Plenum.

Scherer, K. R. (2005):

"What are emotions?

And how can they be measured?".

Social Science Information,

Vol. 44, pp. $695-729$.

Thiel, K. J., and Dretsch, M. N. (2011):

"The basics of the stress response:

A historical context and introduction", in C. D. Conrad (Ed.),

The Handbook of Stress:

Neuropsychological Effects on the Brain, pp. 1-28. Wiley-Blackwell.

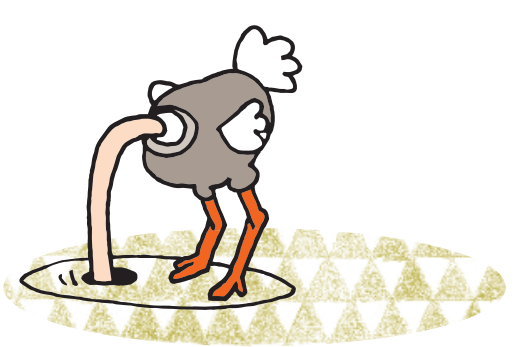

